\title{
Complex-Path Prediction of Resonance-Assisted Tunneling in Mixed Systems
}

\author{
Felix Fritzsch, ${ }^{1,2}$ Arnd Bäcker, ${ }^{1,2}$ Roland Ketzmerick, ${ }^{1,2}$ and Normann Mertig ${ }^{1,2,3}$ \\ ${ }^{1}$ Technische Universität Dresden, Institut für Theoretische Physik and Center for Dynamics, 01062 Dresden, Germany \\ ${ }^{2}$ Max-Planck-Institut für Physik komplexer Systeme, Nöthnitzer Straße 38, 01187 Dresden, Germany \\ ${ }^{3}$ Department of Physics, Tokyo Metropolitan University, Minami-Osawa, Hachioji 192-0397, Japan
}

(Dated: November 7, 2018)

\begin{abstract}
We present a semiclassical prediction of regular-to-chaotic tunneling in systems with a mixed phase space, including the effect of a nonlinear resonance chain. We identify complex paths for direct and resonance-assisted tunneling in the phase space of an integrable approximation with one nonlinear resonance chain. We evaluate the resonance-assisted contribution analytically and give a prediction based on just a few properties of the classical phase space. For the standard map excellent agreement with numerically determined tunneling rates is observed. The results should similarly apply to ionization rates and quality factors.
\end{abstract}

Tunneling through energetic barriers is a textbook paradigm of quantum mechanics. While classically motion is confined to either side of the barrier, wave functions exhibit contributions on both sides. In contrast, nature often exhibits confinement on dynamically disjoint regions of regular and chaotic motion in a mixed phase space, see Fig. 1(a). Here, a classical particle follows a trajectory of regular motion while the correponding wave function admits an exponentially small contribution on the chaotic region. This phenomenon is called dynamical tunneling [1, 2].

Until today dynamical tunneling has emerged in many fields of physics. It determines the vibrational spectrum of molecules [1, ionization rates of atoms in laser fields [3, 4, and chaos-assisted tunneling oscillations [5, 6] in cold atom systems [7, 8. In optics dynamical tunneling is experimentally explored in microwave resonators $9-12$ as well as microlasers 13 20, where it determines the quality factor of lasing modes. Here, a recent experimental breakthrough [12, 17] is the measured enhancement of dynamical tunneling due to nonlinear resonance chains [21, 22].

To reveal the universal features of dynamical tunneling it is extensively studied theoretically [10, 13, 23, 43. mainly in model systems. A central object is the tunneling rate $\gamma_{m}$, which describes the transition from a state on the $m$ th quantizing torus of the regular region into the chaotic sea. Qualitatively $\gamma_{m}$ can be understood from the theory of resonance-assisted tunneling [21, 22, 27, 44, see dashed line in Fig. 1(b): On average $\gamma_{m}$ decreases exponentially for decreasing wavelength or decreasing effective Planck constant, i.e. Plancks constant scaled to some typical action of the system. In addition a drastic enhancement of $\gamma_{m}$ is observed for some values of $h$. This is due to resonant coupling of regular states, induced by a nonlinear resonance chain [45] within the regular region, see Fig. 11(a).

Despite extensive effort an intuitive, trajectory-based picture of dynamical tunneling from regular to chaotic regions, including the effect of nonlinear resonances is not yet available. Semiclassical theories exist only for time- domain quantities 23,24$]$, cases when resonances are irrelevant [39, and near-integrable systems [22, 46, 47]. On the other hand, quantitatively accurate predictions of $\gamma_{m}$, 43, 48 explicitely require integrable approximations [31, 37, 38, 49] which needs some numerical effort.

In this paper we establish an intuitive, semiclassical,
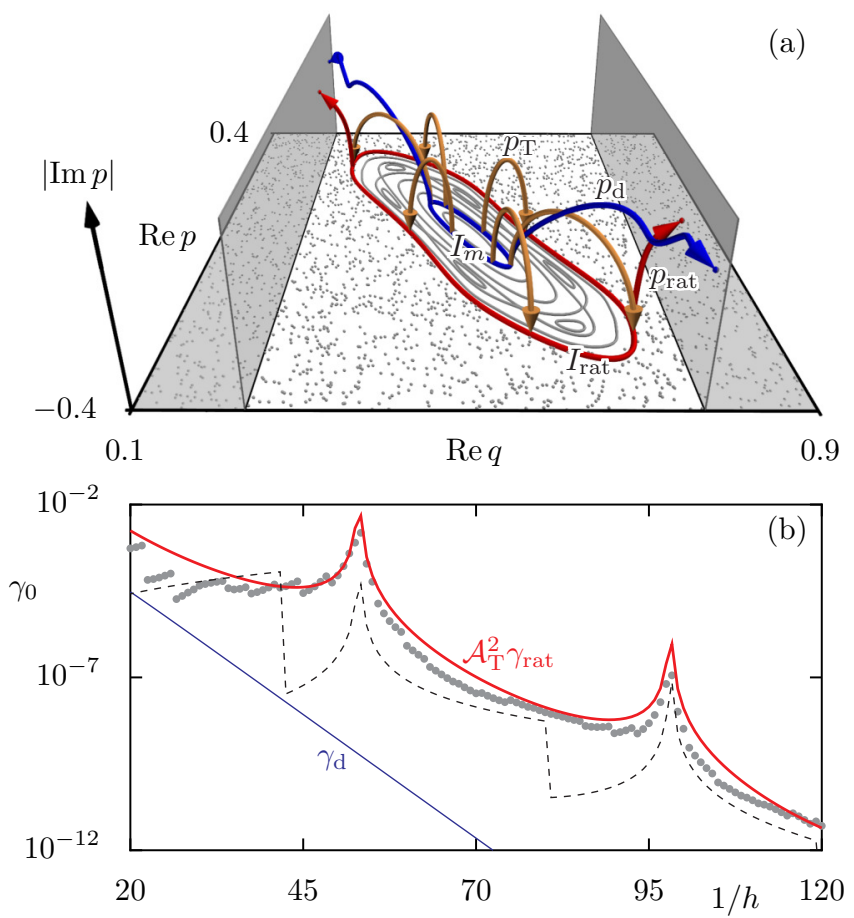

FIG. 1. (color online) (a) Phase space of the standard map for $\kappa=3.4$ with regular tori (thin [gray] lines), and chaotic orbits ([gray] dots). The shaded areas indicate a leaky region in the chaotic part (see text). The real tori and complex paths of the integrable approximation (thick, labeled lines and arrows) connect the regular and the chaotic region. (b) Tunneling rate $\gamma_{0}$ vs. inverse effective Planck constant $1 / h$. Numerically obtained rates (dots) are in excellent agreement with an analytic evaluation of the resonance-assisted contribution $\mathcal{A}_{\mathrm{T}}^{2} \gamma_{\mathrm{rat}}$. Also shown are $\gamma_{\mathrm{d}}$ and the perturbative result 44] (dashed line). 
trajectory-based picture of resonance-assisted regular-tochaotic tunneling in systems with a mixed phase space. It results in a closed-form, analytic formula for tunneling rates $\gamma_{m}$. Our approach gives excellent agreement with numerical results for the standard map, which outperforms the perturbative approach see Fig. 1 (b). Since our final formula requires just a few properties of the classical phase space rather than the construction of a full integrable approximation, it should also allow for estimating ionization rates and quality factors and be helpful, e.g., for designing experimental setups.

Overview - Our method is based on a semiclassical evaluation of a recently developed non-perturbative prediction of $\gamma_{m}$ 43. At its heart is an integrable approximation of the regular region, which includes the relevant nonlinear resonance chain 49 . In that, we justify and generalize the use of semiclassical techniques developed for near-integrable systems [22, 46, 47] in the wider class of generic systems with a mixed phase space. In particular, the integrable approximation overcomes the separation of regular and chaotic motion and allows for connecting real tori to the chaotic region via tunneling paths through complexified phase space. This gives the tunneling rate

$$
\gamma_{m}=\gamma_{\mathrm{d}}+\mathcal{A}_{\mathrm{T}}^{2} \gamma_{\mathrm{rat}}
$$

which is composed of a direct contribution $\gamma_{\mathrm{d}}$ and a resonance-assisted contribution $\mathcal{A}_{\mathrm{T}}^{2} \gamma_{\text {rat }}$, see (blue and red) lines in Fig. 1(b). Figure 1(a) gives an illustration of the phase-space structures contributing to Eq. (1):

(i) Quantizing torus $I_{m}$ and direct tunneling paths $p_{\mathrm{d}}$ : The quantizing torus $I_{m}$, associated with the $m$ th regular state, gives rise to tunneling paths $p_{\mathrm{d}}(q)$ with complex momentum emanating from the turning points of $I_{m}$. See (blue) inner ring and arrows, respectively. They connect $I_{m}$ with the chaotic sea and determine the direct tunneling rate $\gamma_{\mathrm{d}}$, Eq. (7).

(ii) Partner torus $I_{\text {rat }}$ and resonance-assisted tunneling paths $p_{\text {rat }}$ : A partner torus with action $I_{\text {rat }}$ on the opposite side of the nonlinear resonance is connected with the chaotic sea by complex tunneling paths $p_{\text {rat }}(q)$, see (red) outer ring and arrows. They lead to the resonanceassisted tunneling rate $\gamma_{\text {rat }}$, Eq. (7).

(iii) Tunneling paths $p_{\mathrm{T}}$ : The tori $I_{m}$ and $I_{\text {rat }}$ are connected by complex paths $p_{\mathrm{T}}(q)$ bridging the resonance, see (orange) arrows. They determine the tunneling amplitude $\mathcal{A}_{\mathrm{T}}$, Eq. (6).

Basic setting - We derive our results for kicked one-dimensional Hamiltonians $H(q, p, t)=T(p)+$ $V(q) \sum_{n} \delta(t-n)$. For illustrations we use $T(p)=p^{2} / 2$ and $V(q)=\kappa \cos (2 \pi q) /\left(4 \pi^{2}\right)$, giving the paradigmatic standard map [50, which is widely used to study tunneling phenomena [27, 31, 38, 43, 44]. At $\kappa=3.4$ the corresponding stroboscobic Poincaré map exhibits a mixed phase space as shown in Fig. 1(a) with regions of regular motion (thin [gray] lines) and chaotic motion (dots). It is governed by a regular island containing a prominent $r: s=6: 2$ nonlinear resonance chain and a surrounding chaotic sea. Quantum mechanically the dynamics is given by the unitary time-evolution operator $\widehat{U}=\exp (-\mathrm{i} V(\hat{q}) / \hbar) \exp (-\mathrm{i} T(\hat{p}) / \hbar))$. By introducing a leaky region $\mathcal{L}$ (shaded areas in Fig. 1(a)) close to the regular-chaotic border we compute tunneling rates $\gamma_{m}$ as discussed in Ref. [43. We focus on the ground state $(m=0)$ which localizes on the innermost quantizing torus of the regular island. Its tunneling rate is shown in Fig. 1. (b) (dots). Note that higher excited states $(m>0)$ show the same qualitative features.

Integrable approximation - The key tool for deriving our prediction is an integrable approximation. It is a one degree of freedom time-independent Hamiltonian, which resembles the regular dynamics of the original system [49. It is based on the universal description of the classical dynamics in the vicinity of a $r: s$ resonance by the pendulum Hamiltonian 21, 22, 46, 49.

$$
\mathcal{H}_{r: s}(\theta, I)=\mathcal{H}_{0}(I)+2 V_{r: s}\left(\frac{I}{I_{r: s}}\right)^{r / 2} \cos (r \theta),
$$

using action-angle coordinates of $\mathcal{H}_{0}(I)$. It is determined by the frequencies $\omega_{0}(I)$ of tori in the co-rotating frame of the resonance, as $\omega_{0}(I)=\partial_{I} \mathcal{H}_{0}(I)$ [49], where $\mathcal{H}_{0}(I) \approx\left(I-I_{r: s}\right)^{2} /\left(2 M_{r: s}\right)$ close to the resonant torus $I_{r: s}$. The quantities $I_{r: s}, V_{r: s}$, and $M_{r: s}$ can be computed from the position and the size of the resonance chain and the linearized dynamics of its central orbit [27, 49]. The phase space is depicted by thin [gray] lines in Fig. 2 . Via a canonical transformation $T$ the Hamiltonian Eq. (2) is mapped onto the phase space of the standard map giving $H_{r: s}(q, p)=\mathcal{H}_{r: s}\left(T^{-1}(q, p)\right)$ [49]. By quantizing and diagonalizing $H_{r: s}$ its eigenstates $\psi_{m}$ yield the tunneling rate 43 ]

$$
\gamma_{m}=\int_{\mathcal{L}}\left|\psi_{m}(q)\right|^{2} \mathrm{~d} q
$$

via the probability of $\psi_{m}(q)$ on the leaky region $\mathcal{L}$, which we evaluate semiclassically in the following. As discussed in Ref [43, Eq. (3) provides a good prediction of the tunneling rate $\gamma_{m}$ because $\psi_{m}(q)$ approximates the corresponding state of the mixed system on the regular region and further provides a sufficiently accurate extension into the regular-chaotic border region, which dominates Eq. (3).

WKB construction - Using WKB-techniques [51, 52. we now construct the state $\psi_{m}$ within the integrable approximation $H_{r: s}(q, p)$. This extends the semiclassical methods developed for integrable systems [47] to systems with a mixed phase space. Note that, the use of the integrable approximation solves the problem of natural boundaries 53,54 . Thus the integrable approximation is the key for connecting regular and chaotic motion quantum mechanically. 
Following [52, the wave function is constructed from generalized plane waves with locally adapted momentum, Eq. (5). This requires the solutions $p_{\alpha}(q)$ of the equation $E_{m}=H_{r: s}\left(q, p_{\alpha}(q)\right)$, as depicted in Fig. 1(a). Here, $E_{m}$ is the energy of the wave function $\psi_{m}(q)$ obtained from EBK quantization. The position coordinate $q$ is real. The real solutions $p_{\alpha}(q)$ describe the oscillatory part of the wave function in classically allowed regions. The complex solutions $p_{\alpha}(q)$ describe the exponentially decreasing tunneling tails of the wave function in classically forbidden regions. In particular, they describe $\psi_{m}(q)$ in the leaky region, as required by Eq. (3).

Specifically, for resonances the geometry of paths gives the semiclassical wave function as a superposition

$$
\psi_{m}(q)=\psi_{\mathrm{d}}(q)+\mathcal{A}_{\mathrm{T}} \psi_{\mathrm{rat}}(q) .
$$

Here, (i) $\psi_{\mathrm{d}}(q)$ is the direct wave function, (ii) $\psi_{\text {rat }}(q)$ is the resonant wave function, and (iii) $\mathcal{A}_{\mathrm{T}}$ is the tunneling amplitude. We now explain this in more detail:

(i) $\psi_{\mathrm{d}}(q)$ describes the wave function along the quantizing torus $I_{m}$ in the classically allowed region of energy $E_{m} \approx \mathcal{H}_{0}\left(I_{m}\right)$, which is obtained from EBK quantization of the torus $I_{m}=\hbar(m+1 / 2)$. Using Airy-type connections 52 this wave function is extended into the classically forbidden region along the paths $\left(q, p_{\alpha}(q)\right.$ ) (with $\alpha=$ d), see Fig. 1 (a), as

$$
\psi_{\alpha}(q)=\left|\frac{\omega_{0}\left(I_{m}\right)}{2 \pi \partial_{p} H_{r: s}\left(q, p_{\alpha}(q)\right)}\right|^{1 / 2} \exp \left(\frac{\mathrm{i}}{\hbar} \int^{q} p_{\alpha}(\tilde{q}) \mathrm{d} \tilde{q}\right) .
$$

Here, $\omega_{0}\left(I_{m}\right) /(2 \pi)$ accounts for global normalization of the wave function, while $1 /\left|\partial_{p} H_{r: s}\left(q, p_{\alpha}(q)\right)\right|$ is the classical probability along $p_{\alpha}(q)$. The complex action $\int p_{\alpha}(q) \mathrm{d} q$, for which the lower limit is one of the turning points on the torus $I_{m}$, describes direct tunneling into the leaky region.

(ii) Due to the presence of the nonlinear resonance there is an additional real solution $I_{\text {rat }}$ with energy $E_{m}$ on the opposite side of the resonance chain. Along this torus $I_{\text {rat }}$ we construct the wave function $\psi_{\text {rat }}(q)$. In particular, the tunneling tails associated with the solutions $p_{\text {rat }}(q)$ emanating from $I_{\text {rat }}$ and connecting to the chaotic part of phase space, see Fig. 1(a), also obey Eq. (5) with $\alpha=$ rat. Note that $\omega_{0}\left(I_{m}\right) /(2 \pi)$ in Eq. (5) must be kept for normalization. The lower limit of the action integral is one of the turning points on $I_{\text {rat }}$.

(iii) Finally, the tunneling amplitude is given by 22 .

$$
\mathcal{A}_{\mathrm{T}}=\left|2 \sin \left(\frac{\pi}{r \hbar}\left[I_{\text {rat }}-I_{m}\right]\right)\right|^{-1} \exp \left(-\frac{\sigma}{\hbar}\right),
$$

where $\sigma=\operatorname{Im} \int p_{\mathrm{T}}(q) \mathrm{d} q$ is the imaginary part of the action of any path $p_{\mathrm{T}}(q)$ connecting $I_{m}$ to $I_{\text {rat }}$. In particular, since there is no solution $p_{\alpha}(q)$ connecting $I_{m}$ and $I_{\text {rat }}$ along real positions, these paths are only sketched schematically in Fig. 11(a). Note that this evaluation of $\mathcal{A}_{\mathrm{T}}$ based on paths with complex position is formally beyond the WKB-construction used here. It has been introduced and successfully applied for near-integrable systems in Ref. 47. Further note that complex solutions which do not connect to a real torus are neglected.

To summarize our construction, the wave functions $\psi_{\mathrm{d}}(q)$ and $\psi_{\text {rat }}(q)$, Eq. (5), together with the tunneling amplitude, Eq. (6), give the wave function $\psi_{m}(q)$, Eq. (4). Inserting $\psi_{m}(q)$ into Eq. (3) and neglecting interference allows for evaluating the integral in Eq. (3) independently for $\psi_{\mathrm{d}}(q)$ and $\psi_{\text {rat }}(q)$. For solving these integrals we linearize the action integral in Eq. (5) around the boundary of the leaky region at $q_{\mathcal{L}}$. We further account for the symmetry of the standard map with respect to the central fixed point. This gives (i) the direct $(\alpha=\mathrm{d})$ and (ii) the resonance-assisted ( $\alpha=$ rat) tunneling rate as

$$
\gamma_{\alpha}=\frac{\hbar}{\operatorname{Im} p_{\alpha}\left(q_{\mathcal{L}}\right)}\left|\psi_{\alpha}\left(q_{\mathcal{L}}\right)\right|^{2}
$$

i. e., each tunneling rate is given by the value of the normalized WKB wave function at the boundary $q_{\mathcal{L}}$ of the leaky region.

This construction constitutes our first main result. $\mathrm{Nu}-$ merical evaluation of the semiclassically obtained tunneling rates shows excellent agreement with numerical obtained tunneling rates (not shown). This generalizes previous work, Refs. [22, 46, 47, to the much larger class of mixed system, based on the powerful tool of integrable approximations. It further provides a basis for a fully analytic prediction, which no longer requires constructing integrable approximations explicitely: Namely, we observe that for the standard map at $\kappa=3.4$ (and other examples) the resonance-assisted contribution dominates the semiclassically predicted decay rates for all values of the effective Planck constant. In general, one can expect $\gamma_{m}=\mathcal{A}_{\mathrm{T}}^{2} \gamma_{\text {rat }}$, whenever the resonance is sufficiently large, i.e. roughly speaking when it is visible within the regular region. The converse, that $\gamma_{m}$ is dominated by the direct tunneling rate $\gamma_{\mathrm{d}}$, may occur for small values of $1 / h$ or if the resonance is extremely small.

Analytic result $\gamma_{m} \approx \mathcal{A}_{\mathrm{T}}^{2} \gamma_{\text {rat }}$ - In the following we derive an analytic formula which evaluates the dominating term $\gamma_{m}=\mathcal{A}_{\mathrm{T}}^{2} \gamma_{\text {rat }}$ based on just a few properties of the classical phase space. To this end we use the pendulum Hamiltonian $\mathcal{H}_{r: s}(\theta, I)$, Eq. (2), in action-angle coordinates of $\mathcal{H}_{0}(I)$ and the action representation $\psi_{m}(I)$ of the WKB wave function, respectively. This extends the WKB construction presented in Ref. 22] to the Hamiltonian (2). In this context the main novelty is to account both for the action dependence of the resonance term proportional to $V_{r: s}$ and to obtain a closed form expression for $\gamma_{\text {rat }}$.

As a first approximation we extend the leaky region to all chaotic trajectories as $I>I_{\mathcal{L}}$ (shaded area in Fig. 2). In order to account for sticky motion, we choose $I_{\mathcal{L}}$ such that $2 \pi I_{\mathcal{L}}$ is the area enclosing the regular region enlarged up to the most relevant partial barrier [27, 44]. 


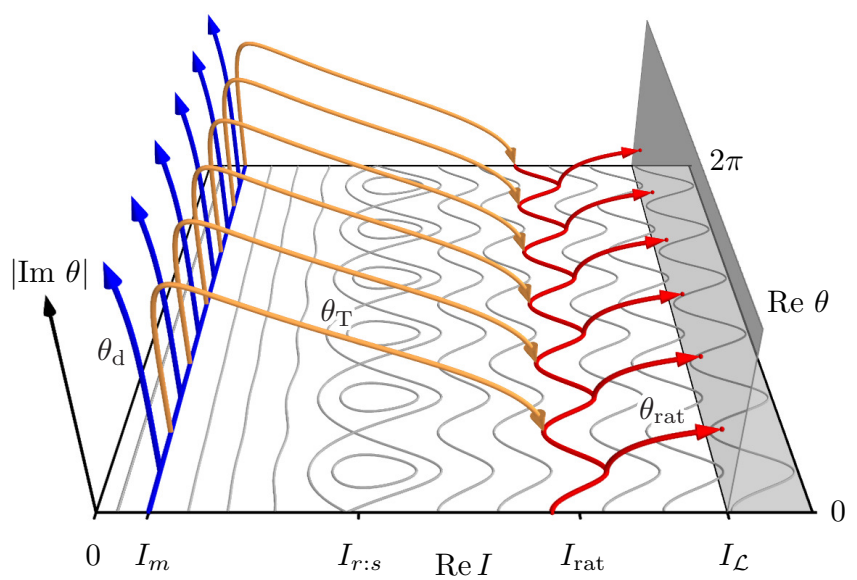

FIG. 2. (color online) Phase space of $\mathcal{H}_{r: s}$ (thin [gray] lines) and leaky region $\mathcal{L}$ (shaded area). Real tori an complex paths (thick lines and arrows) are labeled in the figure.

While the basic features of $\gamma_{m}$ are preserved upon changing the leaky region, it is worth noting that its details might change roughly up to two orders of magnitude [43]. This constitutes the main error of our prediction.

To construct the WKB wave function the classical phase-space structures $\theta_{\alpha}(I)$ fulfilling $\mathcal{H}_{r: s}(\theta, I)=E_{m}$ for real actions are required. They are depicted in Fig. 2 and obey $\cos (r \theta)=\varphi(I)$, where

$$
\varphi(I)=\left(\frac{I_{r: s}}{I}\right)^{r / 2} \frac{E_{m}-\mathcal{H}_{0}(I)}{2 V_{r: s}} .
$$

Real solutions correspond to the tori oscillating around $I_{m}$ and $I_{\text {rat }}$, i.e. the classically allowed regions ([blue and red] thick lines) on opposite sides of the resonance chain at $I_{r: s}$. We have $I_{m}=\hbar(m+1 / 2)$ and a reasonable approximation of $I_{\text {rat }}$ is obtained from $I_{\text {rat }} \approx 2 I_{r: s}-$ $I_{m}+\left[E_{m}-\mathcal{H}_{0}\left(2 I_{r: s}-I_{m}\right)\right] / \omega_{0}\left(2 I_{r: s}-I_{m}\right)$. The torus $I_{m}$ is accompanied by complex paths $\theta_{\mathrm{d}}(I)$ ([blue] arrows) which emanate from turning points with $I<I_{m}$ and diverge at $I=0$. Furthermore, there are tunneling paths $\theta_{\mathrm{T}}(I)$ ([orange] arrows) with imaginary part $\operatorname{Im} \theta_{\mathrm{T}}(I)=$ $\operatorname{arccosh}(|\varphi(I)|) / r$ attached to turning points with $I>$ $I_{m}$ bridging the resonance towards $I_{\text {rat }}$. Finally, there are complex paths $\theta_{\text {rat }}(I)$ ([red] arrows) emanating from turning points with $I>I_{\text {rat }}$ on the partner torus. They have imaginary part $\operatorname{Im} \theta_{\text {rat }}(I)=\operatorname{arccosh}(|\varphi(I)|) / r$ as well and connect $I_{\text {rat }}$ with the leaky region.

Using Eq. (5), with accordingly interchanged phasespace coordinates, local WKB wave functions can be constructed from these paths. Again a global construction of $\psi_{m}(I)$ is obtained by using Airy-type connections at classical turning points 22,52 . In action-angle coordinates the torus $I_{m}$ is not directly connected with the leaky region. Thus, for $\psi_{m}(I)$ there is neither a direct contribution to the WKB wave function within $\mathcal{L}$ nor a direct tunneling rate $\gamma_{\mathrm{d}}$ involved in this construction. Consequently, one has $\psi_{m}(I)=\mathcal{A}_{\mathrm{T}} \psi_{\text {rat }}(I)$ within $\mathcal{L}$. As the tunneling amplitude $\mathcal{A}_{\mathrm{T}}$, Eq. (6), is canonically invariant, it can be computed in action-angle coordinates as well, requiring the evaluation of $\sigma=\operatorname{Im} \int \theta_{\mathrm{T}} \mathrm{d} I$ from $I_{m}$ to $I_{\text {rat }}$. By approximating $\operatorname{Im} \theta_{\mathrm{T}}(I) \approx \ln (2|\varphi(I)|) / r$, which is justified if $V_{r: s} \ll E_{m}$, and using only the quadratic part of $\mathcal{H}_{0}(I)$, we find

$$
\begin{aligned}
\sigma= & \frac{I_{\mathrm{rat}}-I_{m}}{r} \ln \left(\frac{\left(I_{\mathrm{rat}}-I_{m}\right)^{2}}{2 e^{2} M_{r: s} V_{r: s}}\right) \\
& +\frac{I_{m}}{2} \ln \left(\frac{I_{m}}{e I_{r: s}}\right)-\frac{I_{\mathrm{rat}}}{2} \ln \left(\frac{I_{\mathrm{rat}}}{e I_{r: s}}\right),
\end{aligned}
$$

which inserted in Eq. (6) constitutes the first part of our analytic expression. Note that the first term coincides with the results obtained for a simpler pendulum model in Ref. 22] while the remaining terms are related to the action dependence of the resonance term proportional to $V_{r: s}$ in Eq. (2).

We proceed by computing $\gamma_{\text {rat }}$ by Eq. (3) from the WKB wave function $\psi_{\text {rat }}(I)$ and its probability inside the leaky region. The WKB wave function $\psi_{\text {rat }}(I)$ is associated with $\theta_{\text {rat }}(I)$ and computed analogously to Eq. (5) using action-angle coordinates. Linearizing the tunneling action occurring in the exponential in Eq. (5) around $I_{\mathcal{L}}$ then gives

$$
\gamma_{\text {rat }}=\frac{r \hbar}{2 \ln \left(2\left|\varphi\left(I_{\mathcal{L}}\right)\right|\right)}\left|\psi_{\text {rat }}\left(I_{\mathcal{L}}\right)\right|^{2},
$$

which is in close analogy with Eq. (7). Again the resonance-assisted tunneling rate is determined by the normalized WKB wave function

$$
\left|\psi_{\text {rat }}\left(I_{\mathcal{L}}\right)\right|^{2}=\left|\frac{\omega_{0}\left(I_{m}\right)}{2 r \pi\left(E_{m}-\mathcal{H}_{0}\left(I_{\mathcal{L}}\right)\right)}\right| \exp \left(-\frac{2}{\hbar} \mathcal{S}_{\text {rat }}\right)
$$

at the boundary of the leaky region. The tunneling action $\mathcal{S}_{\text {rat }}=\operatorname{Im} \int \theta_{\text {rat }} \mathrm{d} I$ from $I_{\text {rat }}$ to $I_{\mathcal{L}}$ is evaluated similarly as Eq. (9) leading to

$$
\begin{aligned}
\mathcal{S}_{\text {rat }}= & \frac{I_{\mathcal{L}}-I_{\text {rat }}}{r} \ln \left(\frac{\left(I_{\mathcal{L}}-I_{\text {rat }}\right)\left(I_{\mathcal{L}}-I_{m}\right)}{2 e^{2} M_{r: s} V_{r: s}}\right) \\
& +\frac{I_{\text {rat }}}{2} \ln \left(\frac{I_{\text {rat }}}{e I_{r: s}}\right)-\frac{I_{\mathcal{L}}}{2} \ln \left(\frac{I_{\mathcal{L}}}{e I_{r: s}}\right) \\
& +\frac{I_{\text {rat }}-I_{m}}{r} \ln \left(\frac{I_{\mathcal{L}}-I_{m}}{I_{\text {rat }}-I_{m}}\right),
\end{aligned}
$$

which concludes the computation of $\gamma_{\text {rat }}$.

Discussion - The evaluation of $\gamma_{m} \approx \mathcal{A}_{\mathrm{T}}^{2} \gamma_{\text {rat }}$, based on the analytic expressions, Eqs. (6) and (9) for $\mathcal{A}_{\mathrm{T}}$ and Eqs. 111-12 for $\gamma_{\text {rat }}$, requires just a few classical quantities, namely $I_{r: s}, V_{r: s}$, and $M_{r: s}$ as well as the frequencies $\omega_{0}(I)$. This analytic prediction is in excellent agreement with numerically obtained rates, see Fig. 1(b). The resonance peaks originate from the divergence of the prefactor $\mathcal{A}_{\mathrm{T}}$, Eq. (6), i. e., they appear whenever $I_{\text {rat }}$ fulfills a 
quantization condition $I_{\text {rat }}=\hbar(m+l r+1 / 2)$. In particular, at the resonance peak a hybridization between states associated with the $m$ th and $(m+l r)$ th quantizing torus occurs. This is the same resonance condition as obtained from perturbation theory 21. In contrast, away from the resonance peak the tori $I_{m}$ and $I_{\text {rat }}$ are still energetically degenerate. However, since $I_{\text {rat }}$ does not fulfill a quantization condition there is no associated quantum state. This is different from the perturbative framework, where several quantizing tori of different energy contribute to the final prediction. Finally, in contrast to perturbation theory, our result is dominated by a single term for all values of the effective Planck constant. Its overall exponential decay is dominated by the first term of the action $\sigma$, Eq. (9). Hence, the slope of the exponential decay, as depicted in Fig 1(b), is roughly proportional to the width of the dynamical tunneling barrier $I_{\text {rat }}-I_{m}$.

Summary and outlook - We derive a trajectorybased, semiclassical prediction of resonance-assisted regular-to-chaotic tunneling rates in systems with a mixed phase space. To this end we generalize the semiclassical picture valid in near-integrable systems to the larger class of systems with a mixed phase space, based on integrable approximations which include the relevant resonance chain. From this result we find a direct and a resonce-assisted contribution. The latter usually dominates the whole experimentally accessible regime of large tunneling rates. For this resonance-assisted contribution we derive a closed-form analytic expression which depends on just a few properties of the classical phase space. In particular, this expression does not require the explicit construction of integrable approximations. Testing our analytic result for the paradigmatic example of the standard map we find excellent agreement with numerically determined tunneling rates. We expect that our result should also apply to ionization rates and quality factors.

We gratefully acknowledge fruitful discussions with Yasutaka Hanada, Kensuke Ikeda, Julius Kullig, Clemens Löbner, Steffen Löck, Amaury Mouchet, Peter Schlagheck, and Akira Shudo. We acknowledge support by the Deutsche Forschungsgemeinschaft (DFG) Grant No. BA 1973/4-1. N.M. acknowledges successive support by JSPS (Japan) Grant No. PE 14701 and Deutsche Forschungsgemeinschaft (DFG) Grant No. ME 4587/1-1. All 3D visualizations were created using MAYAVI [55].

[1] M. J. Davis and E. J. Heller, Quantum dynamical tunneling in bound states, J. Chem. Phys. 75, 246 (1981).

[2] S. Keshavamurthy and P. Schlagheck, Dynamical Tunneling: Theory and Experiment, Taylor \& Francis, Boca Raton (2011).

[3] S. Wimberger, P. Schlagheck, C. Eltschka, and A. Buch- leitner, Resonance-assisted decay of nondispersive wave packets, Phys. Rev. Lett. 97, 043001 (2006).

[4] J. Zakrzewski, D. Delande, and A. Buchleitner, Ionization via chaos assisted tunneling, Phys. Rev. E 57, 1458 (1998).

[5] W. A. Lin and L. E. Ballentine, Quantum tunneling and chaos in a driven anharmonic oscillator, Phys. Rev. Lett. 65, 2927 (1990).

[6] O. Bohigas, S. Tomsovic, and D. Ullmo, Manifestations of classical phase space structures in quantum mechanics, Phys. Rep. 223, 43 (1993).

[7] W. K. Hensinger et al., Dynamical tunnelling of ultracold atoms, Nature 412, 52 (2001).

[8] D. A. Steck, W. H. Oskay, and M. G. Raizen, Observation of chaos-assisted tunneling between islands of stability, Science 293, 274 (2001).

[9] C. Dembowski, H.-D. Gräf, A. Heine, R. Hofferbert, H. Rehfeld, and A. Richter, First experimental evidence for chaos-assisted tunneling in a microwave annular billiard, Phys. Rev. Lett. 84, 867 (2000).

[10] A. Bäcker, R. Ketzmerick, S. Löck, M. Robnik, G. Vidmar, R. Höhmann, U. Kuhl, and H.-J. Stöckmann, Dynamical tunneling in mushroom billiards, Phys. Rev. Lett. 100, 174103 (2008).

[11] B. Dietz, T. Guhr, B. Gutkin, M. Miski-Oglu, and A. Richter, Spectral properties and dynamical tunneling in constant-width billiards, Phys. Rev. E 90, 022903 (2014).

[12] S. Gehler, S. Löck, S. Shinohara, A. Bäcker, R. Ketzmerick, U. Kuhl, and H.-J. Stöckmann, Experimental observation of resonance-assisted tunneling, Phys. Rev. Lett. 115, 104101 (2015).

[13] V. A. Podolskiy and E. E. Narimanov, Chaos-assisted tunneling in dielectric microcavities, Opt. Lett. 30, 474 (2005).

[14] S. Shinohara, T. Harayama, T. Fukushima, M. Hentschel, T. Sasaki, and E. E. Narimanov, Chaos-assisted directional light emission from microcavity lasers, Phys. Rev. Lett. 104, 163902 (2010).

[15] S. Shinohara, T. Harayama, T. Fukushima, M. Hentschel, S. Sunada, and E. E. Narimanov, Chaos-assisted emission from asymmetric resonant cavity microlasers, Phys. Rev. A 83, 053837 (2011).

[16] J. Yang, S.-B. Lee, S. Moon, S.-Y. Lee, S. W. Kim, T. T. A. Dao, J.-H. Lee, and K. An, Pump-induced dynamical tunneling in a deformed microcavity laser, Phys. Rev. Lett. 104, 243601 (2010).

[17] H. Kwak, Y. Shin, S. Moon, S.-B. Lee, J. Yang, and K. An, Nonlinear resonance-assisted tunneling induced by microcavity deformation, Scientific Reports 5, 9010 (2015).

[18] H. Cao and J. Wiersig, Dielectric microcavities: Model systems for wave chaos and non-hermitian physics, Rev. Mod. Phys. 87, 61 (2015).

[19] C.-H. Yi, H.-H. Yu, J.-W. Lee, and C.-M. Kim, Fermi resonance in optical microcavities, Phys. Rev. E 91, 042903 (2015).

[20] C.-H. Yi, H.-H. Yu, and C.-M. Kim, Resonant torusassisted tunneling, Phys. Rev. E 93, 012201 (2016).

[21] O. Brodier, P. Schlagheck, and D. Ullmo, Resonanceassisted tunneling in near-integrable systems, Phys. Rev. Lett. 87, 064101 (2001).

[22] O. Brodier, P. Schlagheck, and D. Ullmo, Resonanceassisted tunneling, Ann. Phys. (N.Y.) 300, 88 (2002). 
[23] A. Shudo and K. S. Ikeda, Complex classical trajectories and chaotic tunneling, Phys. Rev. Lett. 74, 682 (1995).

[24] A. Shudo and K. S. Ikeda, Chaotic tunneling: A remarkable manifestation of complex classical dynamics in non-integrable quantum phenomena, Physica D 115, 234 (1998).

[25] V. A. Podolskiy and E. E. Narimanov, Semiclassical description of chaos-assisted tunneling, Phys. Rev. Lett. 91, 263601 (2003).

[26] S. Keshavamurthy, Dynamical tunneling in molecules: role of the classical resonances and chaos, J. Chem. Phys. 119, 161 (2003).

[27] C. Eltschka and P. Schlagheck, Resonance- and chaosassisted tunneling in mixed regular-chaotic systems, Phys. Rev. Lett. 94, 014101 (2005).

[28] S. Keshavamurthy, On dynamical tunneling and classical resonances, J. Chem. Phys. 122, 114109 (2005).

[29] M. Sheinman, S. Fishman, I. Guarneri, and L. Rebuzzini, Decay of quantum accelerator modes, Phys. Rev. A 73, 052110 (2006).

[30] S. Keshavamurthy, Dynamical tunneling in molecules: quantum routes to energy flow, Int. Rev. Phys. Chem. 26, 521 (2007).

[31] A. Bäcker, R. Ketzmerick, S. Löck, and L. Schilling, Regular-to-chaotic tunneling rates using a fictitious integrable system, Phys. Rev. Lett. 100, 104101 (2008).

[32] A. Shudo and K. S. Ikeda, Stokes geometry for the quantum hénon map, Nonlinearity 21, 1831 (2008), 00001.

[33] A. Shudo, Y. Ishii, and K. S. Ikeda, Chaos attracts tunneling trajectories: A universal mechanism of chaotic tunneling, Europhys. Lett. 81, 50003 (2008), 00013.

[34] A. Shudo, Y. Ishii, and K. S. Ikeda, Julia sets and chaotic tunneling: I, J. Phys. A 42, 265101 (2009).

[35] A. Shudo, Y. Ishii, and K. S. Ikeda, Julia sets and chaotic tunneling: II, J. Phys. A 42, 265102 (2009).

[36] A. Bäcker, R. Ketzmerick, S. Löck, J. Wiersig, and M. Hentschel, Quality factors and dynamical tunneling in annular microcavities, Phys. Rev. A 79, 063804 (2009).

[37] A. Bäcker, R. Ketzmerick, and S. Löck, Direct regularto-chaotic tunneling rates using the fictitious-integrablesystem approach, Phys. Rev. E 82, 056208 (2010).

[38] S. Löck, A. Bäcker, R. Ketzmerick, and P. Schlagheck, Regular-to-chaotic tunneling rates: From the quantum to the semiclassical regime, Phys. Rev. Lett. 104, 114101 (2010).

[39] N. Mertig, S. Löck, A. Bäcker, R. Ketzmerick, and A. Shudo, Complex paths for regular-to-chaotic tunnelling rates, Europhys. Lett. 102, 10005 (2013).

[40] Y. Hanada, A. Shudo, and K. S. Ikeda, Origin of the enhancement of tunneling probability in the nearly inte- grable system, Phys. Rev. E 91, 042913 (2015).

[41] A. Shudo and K. S. Ikeda, Toward pruning theory of the Stokes geometry for the quantum Hénon map, Nonlinearity 29, 375 (2016).

[42] J. Kullig and J. Wiersig, Frobenius-Perron eigenstates in deformed microdisk cavities: non-Hermitian physics and asymmetric backscattering in ray dynamics, New J. Phys. 18, 015005 (2016).

[43] N. Mertig, J. Kullig, C. Löbner, A. Bäcker, and R. Ketzmerick, Perturbation-free prediction of resonanceassisted tunneling in mixed regular-chaotic systems, arXiv:1607.06477 [nlin.CD] (2016).

[44] P. Schlagheck, A. Mouchet, and D. Ullmo, Resonanceassisted tunneling in mixed regular-chaotic systems, in Dynamical Tunneling: Theory and Experiment, 2, chapter 8, 177.

[45] G. D. Birkhoff, Proof of Poincaré's geometric theorem, Trans. Amer. Math. Soc. 14, 14 (1913).

[46] A. M. Ozorio de Almeida, Tunneling and the semiclassical spectrum for an isolated classical resonance, J. Phys. Chem. 88, 6139 (1984).

[47] J. Le Deunff, A. Mouchet, and P. Schlagheck, Semiclassical description of resonance-assisted tunneling in onedimensional integrable models, Phys. Rev. E 88, 042927 (2013).

[48] C. Löbner, S. Löck, A. Bäcker, and R. Ketzmerick, Integrable approximation of regular islands: The iterative canonical transformation method, Phys. Rev. E 88, 062901 (2013).

[49] J. Kullig, C. Löbner, N. Mertig, A. Bäcker, and R. Ketzmerick, Integrable approximation of regular regions with a nonlinear resonance chain, Phys. Rev. E 90, 052906 (2014).

[50] B. V. Chirikov, A universal instability of manydimensional oscillator systems, Phys. Rep. 52, 263 (1979).

[51] M. V. Berry and K. E. Mount, Semiclassical approximations in wave mechanics, Rep. Prog. Phys. 35, 315 (1972).

[52] S. Creagh, Tunnelling in multidimensional systems, J. Phys. A 27, 4969 (1994).

[53] J. M. Greene and I. C. Percival, Hamiltonian maps in the complex plane, Physica D 3, 530 (1981).

[54] I. C. Percival, Chaotic boundary of a Hamiltonial map, Physica D 6, 67 (1982).

[55] P. Ramachandran and G. Varoquaux, Mayavi: 3D visualization of scientific data, Comput. Sci. Eng. 13, 40 (2011). 